\title{
A Randomized, Double-Blind, Placebo-Controlled, Global Phase 3 Study of Edasalonexent in Pediatric Patients with Duchenne Muscular Dystrophy: Results of the PolarisDMD Trial
}

Richard S. Finkel ${ }^{\mathrm{a}, *}$, Craig M. McDonald ${ }^{\mathrm{b}}$, H. Lee Sweeney ${ }^{\mathrm{c}}$, Erika Finanger ${ }^{\mathrm{d}}$, Erin Neil Knierbein ${ }^{\mathrm{e}}$, Kathryn R. Wagner ${ }^{f}$, Katherine D. Mathews ${ }^{\mathrm{g}}$, Warren Marks ${ }^{\mathrm{h}}$, Jeffrey Statland ${ }^{\mathrm{i}}$, Jessica Nance ${ }^{\mathrm{j}}$, Hugh J. McMillan ${ }^{\mathrm{k}}$, Gary McCullagh ${ }^{1}$, Cuixia Tian ${ }^{\mathrm{m}}$, Monique M. Ryan ${ }^{\mathrm{n}}$, Declan O’Rourke ${ }^{\mathrm{o}}$, Wolfgang Müller-Felber ${ }^{\mathrm{p}}$, Mar Tulinius ${ }^{\mathrm{q}}$, W. Bryan Burnette ${ }^{\mathrm{r}}$, Cam-Tu Nguyen ${ }^{\mathrm{s}}$,

Kayal Vijayakumart ${ }^{\mathrm{t}}$ Jessika Johannsen ${ }^{\mathrm{u}}$, Han C. Phan ${ }^{\mathrm{v}}$, Michelle Eagle ${ }^{\mathrm{w}}$, James MacDougall ${ }^{\mathrm{x}}$, Maria Mancini ${ }^{\mathrm{x}}$ and Joanne M. Donovan ${ }^{\mathrm{x}}$ (For the PolarisDMD Study Group) a St. Jude Children's Research Hospital, Memphis, TN and Nemours Children's Hospital, Orlando, FL, USA

${ }^{\mathrm{b}}$ University of California at Davis, Sacramento, CA, USA

${ }^{\mathrm{c}}$ University of Florida College of Medicine, Gainesville, FL, USA

${ }^{\mathrm{d}}$ Shriners Hospital for Children, Portland, OR, USA

${ }^{\mathrm{e}}$ University of Michigan, MI, Ann Arbor, MI, USA

${ }^{\mathrm{f}}$ Kennedy Krieger Institute, The Johns Hopkins School of Medicine, Baltimore, MD, USA

$\mathrm{g}$ University of Iowa Carver College of Medicine, Iowa City, IA, USA

${ }^{\mathrm{h}}$ Cook Children's Medical Center, Fort Worth, TX, USA

${ }^{\mathrm{i}}$ University of Kansas Medical Center, Kansas City, KS, USA

jJohns Hopkins University, Baltimore, MD, USA

${ }^{\mathrm{k}}$ Children's Hospital of Eastern Ontario, Ottawa, CA, Canada

${ }^{1}$ Royal Manchester Children's Hospital, UK

${ }^{\mathrm{m}}$ Cincinnati Children's Hospital \& University of Cincinnati, Cincinnati, OH, USA

${ }^{\mathrm{n}}$ Royal Children's Hospital, Melbourne, Australia

${ }^{\circ}$ Children's Health Ireland at Temple Street, Dublin, Ireland

${ }^{\mathrm{p}}$ Dr. v. Haunersches Kinderspital, Munich, Germany

${ }^{\mathrm{q}}$ Queen Silvia Children's Hospital, Gothenburg, Sweden

${ }^{\mathrm{r}}$ Vanderbilt University Medical Center, Nashville, TN, USA

${ }^{\mathrm{s}}$ CHU Sainte-Justine, Montreal, CA, Canada

'Bristol Children's Hospital, Bristol, UK

'University Medical Center Hamburg-Eppendorf, Hamburg, Germany

\footnotetext{
${ }^{*}$ Correspondence to: Richard S. Finkel, MD, St. Jude Children's Research Hospital, Mailstop 760, 262 Danny Thomas Place, Memphis, TN 38120. E-mail: Richard.finkel@stjude.org.
} 
${ }^{\mathrm{v}}$ Rare Disease Research, LLC, Atlanta GA, USA

${ }^{\mathrm{w}}$ Atom International Limited, Newcastle upon Tyne, UK

${ }^{\mathrm{x}}$ Catabasis Pharmaceuticals, Inc., Boston, MA, USA

Pre-press 10 June 2021

\begin{abstract}
.
Background: Edasalonexent (CAT-1004) is an orally-administered novel small molecule drug designed to inhibit NF- $\kappa$ B and potentially reduce inflammation and fibrosis to improve muscle function and thereby slow disease progression and muscle decline in Duchenne muscular dystrophy (DMD).

Objective: This international, randomized $2: 1$, placebo-controlled, phase 3 study in patients $\geq 4-<8$ years old with DMD due to any dystrophin mutation examined the effect of edasalonexent $(100 \mathrm{mg} / \mathrm{kg} / \mathrm{day})$ compared to placebo over $52 \mathrm{weeks}$. Methods: Endpoints were changes in the North Star Ambulatory Assessment (NSAA; primary) and timed function tests (TFTs; secondary). Assessment of health-related function used the Pediatric Outcomes Data Collection tool (PODCI).

Results: One hundred thirty one patients received edasalonexent $(n=88)$ and placebo $(n=43)$. At week 52, differences between edasalonexent and placebo for NSAA total score and TFTs were not statistically significant, although there were consistently less functional declines in the edasalonexent group. A pre-specified analysis by age demonstrated that younger patients $(\leq 6.0$ years) showed more robust and statistically significant differences between edasalonexent and placebo for some assessments. Treatment was well-tolerated and the majority of adverse events were mild, and most commonly involved the gastrointestinal system (primarily diarrhea).

Conclusions: Edasalonexent was generally well-tolerated with a manageable safety profile at the dose of $100 \mathrm{mg} / \mathrm{kg} / \mathrm{day}$. Although edasalonexent did not achieve statistical significance for improvement in primary and secondary functional endpoints for assessment of DMD, subgroup analysis suggested that edasalonexent may slow disease progression if initiated before 6 years of age. (NCT03703882)
\end{abstract}

Keywords: Duchenne muscular dystrophy, NF- $\kappa$ B, edasalonexent, CAT-1004; age effects

\section{INTRODUCTION}

Duchenne muscular dystrophy (DMD) occurs in 1 in 3,500-5000 male births and is one of the most common genetic neuromuscular diseases of childhood [1]. DMD is caused by mutations in the dystrophin $D M D$ gene and is uniformly fatal, with premature death most often a result of cardio-pulmonary complications in early to mid-adulthood [2].

An unmet need remains in DMD for diseasemodifying drugs that are well tolerated and effective regardless of dystrophin mutation, that can be used in combination with drugs directly targeting the dystrophin gene, and that can supplant the use of steroids. While steroids remain the standard of care in DMD, they have considerable side effects when used long-term and particularly at the high doses needed in DMD [3]. Dystrophin is a critical protein linker to the sarcolemma membrane complex, and its deficiency results in progressive symmetric muscle weakness and degeneration with loss of contractile function. Activated NF- $\kappa \mathrm{B}$ is a key pathophysiologic link between the lack of dystrophin and the resulting manifestation and progression of DMD, as demonstrated in animal models of DMD where targeted inhibition of NF- $\kappa$ B activation improved muscle mass and function, fibrosis, inflammation, cardiac pathology and activity $[4,5]$. NF $-\kappa \mathrm{B}$ signaling pathways are activated in skeletal muscles undergoing mechanical stress, (e.g., posterior leg muscles) [6] and contribute to muscle degeneration and suppression of muscle regeneration in DMD [7-11]. In contrast, the muscles of DMD patients exposed to minimal mechanical stress (e.g., gracilis and sartorius muscles) have significantly less pathology [12-14].

Edasalonexent (CAT-1004) is an orally-administered novel small molecule [15] that incorporates the structural elements of two known NF- $\kappa$ B inhibitors, salicylic acid and docosahexaenoic acid (DHA, an omega-3 fatty acid), both of which act on different parts of the NF- $\kappa$ B pathway to provide synergistic and amplified NF- $\kappa$ B inhibition [16-19]. Edasalonexent is stable extracellularly and is enzymatically cleaved intracellularly by the native enzyme fatty acid amide hydrolase. The intracellular hydrolysis of edasalonexent facilitates the simultaneous delivery of salicylic acid and DHA to synergistically inhibit $\mathrm{NF}-\kappa \mathrm{B}$ by reducing both activation in the cytoplasm and its ability to induce nuclear transcription. Edasalonexent has improved potency compared to what 
would be observed with administration of equimolar doses of salicylic acid and DHA individually [15, 20].

In Phase 1 studies in adults, edasalonexent showed rapid and saturable oral absorption with minimal accumulation after multiple doses, and inhibited NF$\kappa \mathrm{B}$ activation pathways [20]. In a multipart phase $1 / 2$ trial in patients 4 to 8 years of age with DMD not on steroids (MoveDMD, NCT02439216), edasalonexent inhibited NF- $\kappa \mathrm{B}$ dependent target genes after one week of dosing [21]. Statistically significant changes in MRI T2 measures known to correlate with disease progression were observed after 12 weeks and multiple subsequent time points, compared with a pretreatment period [22]. In this study there appeared to be clinically meaningful slowing of disease progression during edasalonexent treatment compared to a prior untreated observation period, with improvements in biomarkers of muscle health and inflammation [22]. The MoveDMD study informed the design and dose selection for this phase 3 trial.

This phase 3, international, randomized, placebocontrolled study in pediatric patients with DMD not on steroid therapy examined the safety and efficacy of edasalonexent softgel capsules over 52 weeks.

\section{PATIENTS AND METHODS}

\section{Study participants}

Male patients $\geq 4$ to $<8$ years of age with a diagnosis of DMD based on clinical phenotype, with increased creatine kinase and any $D M D$ mutation known to be associated with a DMD phenotype, were enrolled if they were able to perform the Stand from Supine test without assistance in $\leq 10 \mathrm{sec}-$ onds, the ten-meter run-walk test (10MRWT), the 4-stair climb test, and were able to swallow capsules. Enrolled patients had not used corticosteroids within 24 weeks of screening (due to their secondary effect on NF- $\kappa$ B activity) and did not have prior or ongoing medical conditions that would impair study completion or interpretation of results. Complete inclusion and exclusion criteria are provided in supplementary materials.

Written informed consent was obtained prior to study participation. The study was approved by independent Ethics Committees at all sites and conducted in accordance with the Declaration of Helsinki and Good Clinical Practice guidelines as set forth by the International Conference on Harmonization (ICH) and the U.S. Code of Federal Regulations.

\section{Study design}

PolarisDMD (NCT03703882) was conducted at 37 sites in 8 countries (US, Canada, UK, Ireland, Germany, Sweden, Israel and Australia) between November 2018 and September 2020.

Following a 4-week eligibility and baseline screening period, enrolled patients were randomly assigned $2: 1$ to receive either edasalonexent $100 \mathrm{mg} / \mathrm{kg} /$ day (administered in three divided doses of approximately $33 \mathrm{mg} / \mathrm{kg}$ each) softgel capsules (containing either $100 \mathrm{mg}$ or $250 \mathrm{mg}$ edasalonexent) or matched placebo softgel capsules in double-blind fashion daily for 52 weeks. The number of capsules provided to each patient was based on weight and pre-programmed into the interactive response web system (IRWS). Study drug was orally administered with food containing at least 8 grams of fat, as this was previously shown to augment absorption [20, 21]. As necessary due to COVID travel restrictions, capsules could be shipped to the patient's homes according to local site procedures and secured in temperature-controlled shipments facilitated by a courier.

Randomization was stratified by baseline age ( $\leq 6.0$ years or $>6.0$ years), time to stand from supine ( $\leq 5$ seconds or $>5$ seconds), treatment with eteplirsen (yes or no), and region (North America or Europe/Asia/Australia). Siblings of randomized patients were eligible to participate in the study but were not randomized and were assigned to the same treatment group to prevent patients in the same household from having different treatment assignments. The study design and flow of patients is shown in Fig. 1. At the completion of the study, patients were eligible to enroll in an open label extension trial.

Steroid treatment was not discontinued for the trial, so boys in the trial were those for whom corticosteroid use was not yet suitable or deferred by parent or guardian decision. To minimize the risk to participants, the trial included enrollment criteria that would exclude boys clearly in decline and would include only boys who were not suitable for steroid treatment or whose parents declined such treatment. Investigators were to consider the clinical trajectory of patients as assessed every three months and could recommend transition to steroids if they felt that clinical progression warranted withdrawal and steroid initiation. Additionally, the unblinded safety and efficacy data was reviewed every six months by an independent Data Safety Monitoring Board. 


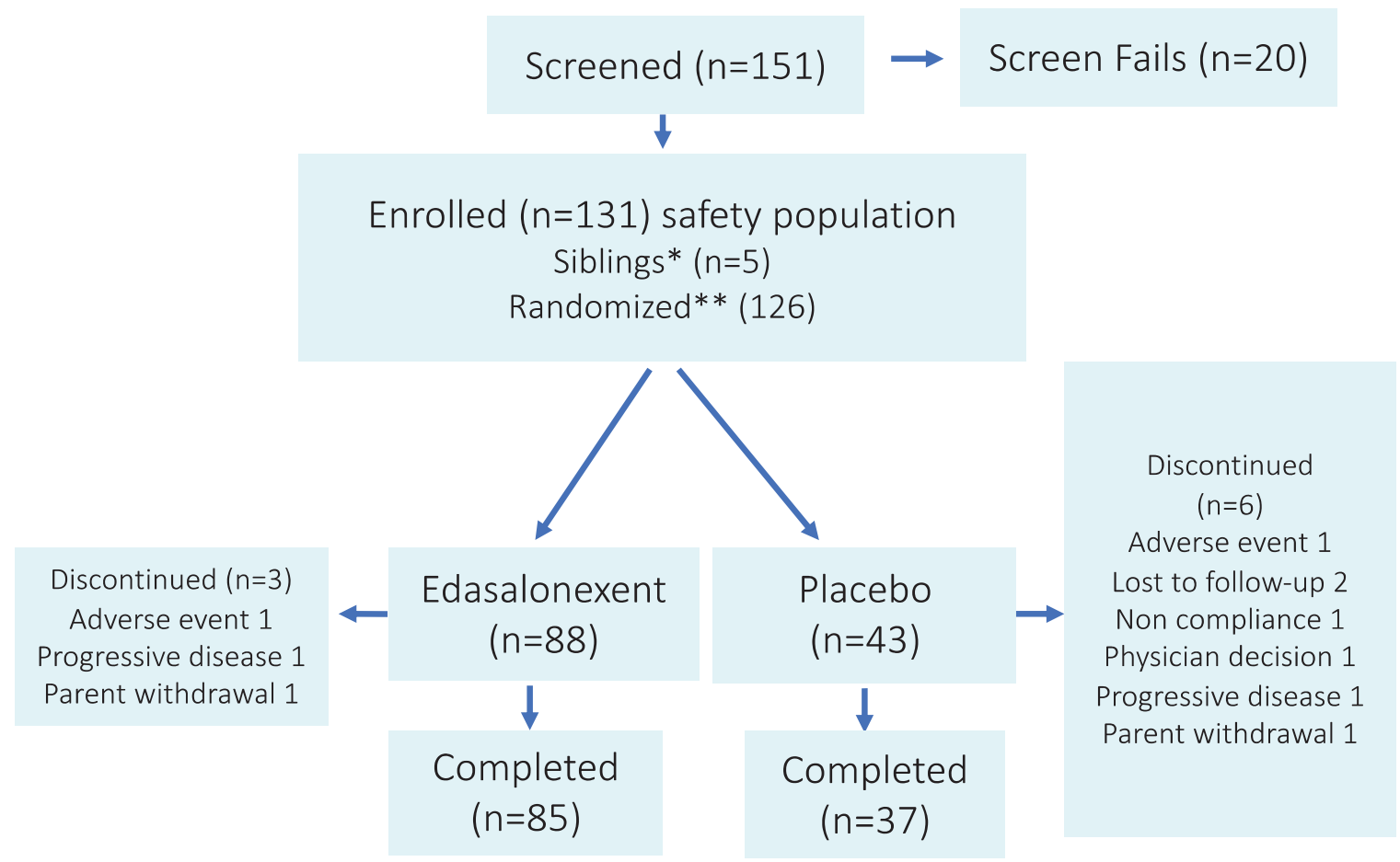

Fig. 1. Study Design and Disposition of Patients. ${ }^{*}$ Siblings of previously randomized patients. ${ }^{* *}$ Among randomized patients, four received eteplirsen and three had no post baseline NSAA, yielding a full analysis set of 119 patients, 81 in the edasalonexent group and 38 in the placebo group.

\section{Study endpoints}

\section{Efficacy}

The primary efficacy assessment was change from baseline in the North Star Ambulatory Assessment (NSAA) total raw score. The NSAA assesses the ability of patients to perform 17 different clinically meaningful activities of motor function, each graded on a 3 point scale (unable-partial-complete achievement), including static skills (standing independently, on heels, and on one leg), dynamic activity (walking, climbing and descending a step, hopping, jumping, and running/walking 10 meters) transitions (rising from supine to sitting, from sitting in a chair to stand, and from floor to standing), and lifting the head in supine[23]. Secondary outcomes included effects of edasalonexent on physical function as assessed by age-appropriate timed motor function tests (10-meter run/walk, climbing 4 stairs, and arising from supine to stand) [21]. Clinical evaluators at all study sites received standardized protocol-specific training and reliability testing with ongoing quality control of assessments by video review and support. Standardized pre-study training, certification and oversight of all clinical evaluator reliability testing was performed by ATOM International Limited (Newcastle upon Tyne, UK).

This trial had completed enrollment and the treatment phase was ongoing at the time of the COVID-19 pandemic and a protocol amendment was approved by IRB/Ethics Committees and implemented to allow flexibility around data collection while maintaining study integrity due to travel restrictions. ATOM International completed feasibility of remote video assessments and prepared standard guidelines for functional assessments collected remotely. Videos were reviewed in the same manner as at Screening/Baseline.

Assessment of health-related function was made using the Pediatric Outcomes Data Collection tool (PODCI) containing 86 questions over 6 scales for upper extremity/physical function, transfer/mobility, sports/physical function, pain/comfort, happiness, and global function and designed to be completed by the parent/caregiver of children aged 10 or younger [24].

The NSAA and timed function tests were conducted at baseline and study weeks 13, 26, 39 and 
52/end of study. The PODCI was conducted at baseline and week 52 (end of study). The 7-point entry item of the Performance of Upper Limb (PUL) scale from 6 through 0, (based on the modified Brooke Upper Extremity Scale), defined the starting functional level and was conducted at baseline and study weeks 26 and 52/end of study.

Pharmacokinetic evaluations assessed the concentration of edasalonexent in plasma at selected time points.

\section{Safety}

Safety and tolerability assessments included treatment emergent and related adverse events monitored throughout treatment and at the follow-up assessment occurring within 2 weeks after the last dose of study drug, and clinical laboratory evaluations (including adrenal function), physical examinations, 12-lead ECGs, and vital signs assessed/conducted at baseline (predose) and throughout treatment. Due to COVID-19, as much as possible was done in a remote capacity if an on-site visit could not be conducted.

Assessments of cardiac autonomic dysfunction, including heart rate variability, were made in patients from a subset of countries using wearable Holter devices at baseline and study weeks 26 and 52/end of study. Lateral thoracolumbar spine radiography scans and DXA scans were performed where feasible at baseline and study week 52/end of study. Cardiac and DXA results will be reported separately.

\section{Statistics}

The primary population for efficacy analysis consisted of all randomized patients who received at least 1 dose of study drug and had at least 1 valid post Baseline NSAA efficacy assessment and excluded patients on eteplirsen at randomization. The safety population consisted of all patients who received at least 1 dose of study drug.

The study was powered based on Phase 2 changes in NSAA compared to an off-treatment control period, with an effect size of 0.625 and a dropout rate of approximately $20 \%$ assumed. The study had approximately $90 \%$ power to show a difference between the treatment groups with a 2-sided type I error rate of 0.05 for a 2.5 point difference in the NSAA Total Score.

The change from baseline in NSAA Total Score was analyzed using a mixed-model repeated-measures (MMRM) analysis of covariance (ANCOVA), implemented using SAS ${ }^{\circledR}$ Proc Mixed. The explana- tory variables in the model were baseline age (continuous variable), baseline time to stand from supine (continuous variable), baseline NSAA score (continuous variable), region (North America or Europe/Asia/Australia), treatment group (edasalonexent or placebo), and visit (a categorical variable). By-visit interaction terms for baseline age, baseline time to stand from supine, baseline NSAA score, region, and treatment group were also included. The primary analysis tested the treatment difference at Week 52 via the MMRM model at the two-sided 0.05 level. Sensitivity analyses were conducted to exclude visits conducted remotely.

Descriptive statistics were calculated for the speed and time to complete timed function tests as well as all change from baseline values by treatment group and time point. Changes from baseline in speed were analyzed using the same MMRM ANCOVA model as for the primary efficacy endpoint with the corresponding baseline speed value used as covariate instead of baseline NSAA value. While all PODCI scales were administered, the PODCI Basic Transfer and Mobility Scale was a prespecified analysis using an ANCOVA with baseline age, baseline time to stand from supine, baseline PODCI Basic Transfer and Mobility score, region, and treatment group as explanatory variables.

For prespecified subgroup analyses, nominal $P$ values are reported without adjustment of multiplicity and statistical significance is defined as nominal $P<0.05$.

\section{RESULTS}

\section{Study participants}

Figure 1 shows the progression of enrolled patients in the study. One hundred fifty one patients were screened and 131 randomized or assigned to edasalonexent $(n=88)$ or placebo $(n=43)$. Twenty patients failed screening because of lack of genetic confirmation $(n=3)$, inability to perform timed function tests adequately $(n=4)$ or stand from supine within 10 seconds $(n=10)$, and/or difficulty swallowing capsules $(n=6)$. Five of the 131 patients were siblings of randomized patients and were not included in randomization. One hundred twenty two patients completed the study and 9 discontinued for reasons shown in Fig. 1. Ninety three percent (113/122) of patients completing the study enrolled in the open label extension trial. Fifteen percent of study assessments were conducted remotely. 
Table 1

Demographic and Baseline Characteristics

\begin{tabular}{|c|c|c|c|}
\hline & $\begin{array}{c}\text { Edasalonexent } \\
100 \mathrm{mg} / \mathrm{kg} \\
(N=88)\end{array}$ & $\begin{array}{l}\text { Placebo } \\
(N=43)\end{array}$ & $\begin{array}{c}\text { Total } \\
(N=131)\end{array}$ \\
\hline Age at Screening (Year), Mean (SD) & $5.65(1.048)$ & $5.77(0.995)$ & $5.69(1.029)$ \\
\hline \multicolumn{4}{|l|}{ Age Group, $n(\%)^{\mathrm{a}}$} \\
\hline$\leq 6.0 \mathrm{yr}$ & $55(62.5)$ & $29(67.4)$ & $84(64.1)$ \\
\hline$>6.0 \mathrm{yr}$ & $33(37.5)$ & $14(32.6)$ & $47(35.9)$ \\
\hline Age at Diagnosis & $3.82(1.70)$ & $3.59(1.94)$ & $3.75(1.78)$ \\
\hline$\leq 6.0 \mathrm{yr}$ & $3.11(1.50)$ & $3.43(1.67)$ & $3.22(1.56)$ \\
\hline$>6.0 \mathrm{yr}$ & $4.96(1.37)$ & $3.93(2.48)$ & $4.68(1.78)$ \\
\hline Age at Symptom Onset & $2.90(1.77)$ & $2.65(1.79)$ & $2.82(1.77)$ \\
\hline$\leq 6.0 \mathrm{yr}$ & $2.41(1.37)$ & $2.78(1.69)$ & $2.54(1.49)$ \\
\hline$>6.0 \mathrm{yr}$ & $3.67(2.06)$ & $2.39(2.01)$ & $3.30(2.11)$ \\
\hline \multicolumn{4}{|l|}{ Race, $n(\%)$} \\
\hline White & $74(84.1)$ & $38(88.4)$ & $112(85.5)$ \\
\hline Black or African American & $4(4.5)$ & $1(2.3)$ & $5(3.8)$ \\
\hline Asian & $3(3.4)$ & $2(4.7)$ & $5(3.8)$ \\
\hline Multiracial & $3(3.4)$ & $1(2.3)$ & $4(3.1)$ \\
\hline Native Hawaiian/Other Pacific Islander & $1(1.1)$ & $0(0.0)$ & $1(0.8)$ \\
\hline Other & $2(2.3)$ & $1(2.3)$ & $3(2.3)$ \\
\hline Unknown & $1(1.1)$ & 0 & $1(0.8)$ \\
\hline \multicolumn{4}{|l|}{ Ethnicity, $n(\%)$} \\
\hline Hispanic or Latino & $14(15.9)$ & $6(14.0)$ & $20(15.3)$ \\
\hline Not Hispanic or Latino & $69(78.4)$ & $35(81.4)$ & $104(79.4)$ \\
\hline Not Reported/unknown & $5(5.7)$ & $2(4.7)$ & $7(5.3)$ \\
\hline \multicolumn{4}{|l|}{ Region, $n(\%)$} \\
\hline North America & $63(71.6)$ & $31(72.1)$ & $94(71.8)$ \\
\hline Europe/Asia/Australia & $25(28.4)$ & $12(27.9)$ & $37(28.2)$ \\
\hline \multicolumn{4}{|l|}{ Treatment with Eteplirsen, $n(\%)$} \\
\hline Yes & $2(2.3)$ & $2(4.7)$ & $4(3.1)$ \\
\hline No & $86(97.7)$ & $41(95.3)$ & $127(96.9)$ \\
\hline \multicolumn{4}{|l|}{ Time to Stand from Supine, $n(\%)$} \\
\hline$\leq 5$ seconds & $48(55.8)$ & $26(60.5)$ & $74(57.4)$ \\
\hline$>5$ seconds & $38(44.2)$ & $17(39.5)$ & $55(42.6)$ \\
\hline Missing, $n(\%)$ & $2(2.3)$ & 0 & $2(1.6)$ \\
\hline Previous corticosteroid use & $2(2.3)$ & $2(4.7)$ & $4(3.1)$ \\
\hline \multicolumn{4}{|l|}{ Mutation type } \\
\hline Deletion & $66(76.1)$ & $31(72.1)$ & $97(74.1)$ \\
\hline Duplication & $18(19.3)$ & $8(18.6)$ & $26(19.1)$ \\
\hline Nonsense & $4(4.5)$ & $4(9.3)$ & $8(6.1)$ \\
\hline
\end{tabular}

Demographics and baseline disease characteristics are shown in Table 1. Mean age was 5.7 years and was similar in the two groups. There were more patients enrolled in the $\leq 6.0$ year age group than in the $>6.0$ group (64\% versus $36 \%$ ). Age at diagnosis and age at symptom onset occurred earlier in the $\leq 6.0$ year age group. The majority of patients were white (112/131, 86\%), not Hispanic/Latino (104/131, $79 \%$ ) and from North America (94/131, 72\%). Four patients were receiving stable eteplirsen treatment $(4 / 131,3 \%)$ for at least 24 weeks prior to enrollment, which was continued during the trial. The population was primarily steroid-naïve $(127 / 131,97 \%)$ with only four patients having used steroids $>24$ weeks prior to study entry. Most patients had deletion mutations (97/131, 74\%). Mean compliance with study drug was $92 \%$ in both the edasalonexent and placebo groups.

At baseline (Table 2), functional assessments showed a reduction in ambulation and endurance that is consistent with previous reports for this age group [21, 24-27]. In general, functional assessment data were similar across groups.

Pharmacokinetic analysis confirmed edasalonexent exposure levels post-dose at week 26 in the edasalonexent group and was consistent with previous studies.

\section{NSAA total score}

The Full Analysis Set included randomized patients not receiving eteplirsen who had post-baseline 
Table 2

Functional Endpoint Assessments for the Full Analysis Population Overall and by Age Group

\begin{tabular}{|c|c|c|c|c|c|c|}
\hline & \multicolumn{2}{|c|}{ Baseline Mean (SD) } & \multicolumn{4}{|c|}{ Week 52 Change from Baseline Mean (SD) } \\
\hline & $\begin{array}{l}\text { Edasalonexent } \\
\text { Overall } n=81 \\
\leq 6.0 \text { yr. } n=51 \\
>6.0 \text { yr. } n=30\end{array}$ & $\begin{array}{c}\text { Placebo } \\
\text { Overall } n=38 \\
\leq 6.0 \text { yr. } n=26 \\
>6.0 \text { yr. } n=12\end{array}$ & Edasalonexent & Placebo & LS Mean $\Delta^{\mathrm{a}}$ & $p$-value \\
\hline \multicolumn{7}{|l|}{ NSAA Score } \\
\hline Overall & $21.5(4.5)$ & $19.5(5.0)$ & $-1.5(4.4)$ & $-1.8(3.8)$ & 0.3 & 0.67 \\
\hline$\leq 6.0$ Years & $20.2(3.6)$ & $18.9(4.9)$ & $0.0(3.3)$ & $-1.0(3.6)$ & 1.4 & 0.08 \\
\hline$>6.0$ Years & $23.9(5.1)$ & $20.8(5.4)$ & $-4.2(4.9)$ & $-3.8(3.7)$ & -0.2 & 0.88 \\
\hline \multicolumn{7}{|c|}{ Stand from Supine Time (sec) } \\
\hline Overall & $5.27(1.89)$ & $5.37(2.05)$ & $1.74(3.35)$ & $3.09(4.81)$ & & \\
\hline$\leq 6.0$ Years & $5.32(1.90)$ & $5.11(1.74)$ & $0.72(2.45)$ & $2.93(4.42)$ & & \\
\hline$>6.0$ Years & $5.18(1.91)$ & $5.93(2.59)$ & $3.70(3.99)$ & $3.46(5.89)$ & & \\
\hline \multicolumn{7}{|l|}{ Speed $\left(\sec ^{-1}\right)$} \\
\hline Overall & $0.211(0.072)$ & $0.212(0.073)$ & $-0.039(0.067)$ & $-0.046(0.062)$ & 0.0011 & 0.92 \\
\hline$\leq 6.0$ Years & $0.208(0.073)$ & $0.218(0.071)$ & $-0.015(0.060)$ & $-0.045(0.059)$ & 0.0271 & 0.046 \\
\hline$>6.0$ Years & $0.221(0.072)$ & $0.199(0.079)$ & $-0.086(0.055)$ & $-0.048(0.071)$ & -0.0350 & 0.12 \\
\hline \multicolumn{7}{|c|}{ 4-Stair Climb Time (sec) } \\
\hline Overall & $4.29(1.86)$ & $4.48(1.68)$ & $1.56(3.91)$ & $2.56(4.85)$ & & \\
\hline$\leq 6.0$ Years & $4.75(1.89)$ & $4.60(1.78)$ & $0.89(3.87)$ & $2.68(5.16)$ & & \\
\hline$>6.0$ Years & $3.51(1.52)$ & $4.21(1.47)$ & $2.93(3.72)$ & $2.30(4.36)$ & & \\
\hline \multicolumn{7}{|l|}{ Speed $\left(\sec ^{-1}\right)$} \\
\hline Overall & $0.271(0.102)$ & $0.254(0.091)$ & $-0.022(0.089)$ & $-0.039(0.074)$ & 0.009 & 0.58 \\
\hline$\leq 6.0$ Years & $0.238(0.080)$ & $0.249(0.092)$ & $0.003(0.077)$ & $-0.038(0.080)$ & 0.044 & 0.021 \\
\hline$>6.0$ Years & $0.332(0.109)$ & $0.265(0.090)$ & $-0.073(0.093)$ & $-0.042(0.060)$ & -0.054 & 0.11 \\
\hline \multicolumn{7}{|c|}{ 10-meter Run/Walk Time (sec) } \\
\hline Overall & $5.71(1.14)$ & $5.84(1.16)$ & $0.32(1.21)$ & $0.64(1.37)$ & & \\
\hline$\leq 6.0$ Years & $5.96(1.17)$ & $5.92(1.12)$ & $0.00(0.94)$ & $0.40(1.19)$ & & \\
\hline$>6.0$ Years & $5.28(0.97)$ & $5.68(1.27)$ & $1.05(1.46)$ & $1.18(1.66)$ & & \\
\hline \multicolumn{7}{|l|}{ Speed $\left(\sec ^{-1}\right)$} \\
\hline Overall & $0.182(0.035)$ & $0.178(0.36)$ & $-0.006(0.030)$ & $-0.009(0.025)$ & -0.0013 & 0.79 \\
\hline$\leq 6.0$ Years & $0.174(0.033)$ & $0.175(0.035)$ & $0.002(0.024)$ & $-0.004(0.024)$ & 0.0048 & 0.41 \\
\hline$>6.0$ Years & $0.198(0.034)$ & $0.184(0.040)$ & $-0.024(0.035)$ & $-0.022(0.025)$ & -0.0051 & 0.67 \\
\hline
\end{tabular}

${ }^{a}$ Least squares (LS) mean difference between groups (edasalonexent minus placebo) without multiplicity adjustments. For prespecified subgroup analyses by age, nominal $p$-values are reported without adjustment of multiplicity and statistical significance is defined as nominal $P<0.05$.

NSAA $(n=119)$. Mean (SD) NSAA total scores at baseline were 21.5 (4.6) and 19.5 (5) in the edasalonexent and placebo groups respectively and decreased (worsened) throughout treatment (Supplemental Figure S1). Mean (SD) total scores at week 52 were 20.1 (5.6) and 17.3 (6.4) in the edasalonexent and placebo groups, respectively. Changes from baseline in NSAA Total Score (Fig. 2A) were negative for the edasalonexent group throughout the treatment period and began to decline in the placebo group after 26 weeks of treatment. At Week 52, the least squares (LS) mean (SE) change from baseline in the NSAA Total Score was $-1.4(0.42)$ in the edasalonexent group and $-1.7(0.61)$ in the placebo group with a LS mean (SE) difference of $0.3(0.73)(p=0.67)$. Results of NSAA sensitivity analyses are included in supplementary material. Sensitivity analysis of change from baseline in NSAA excluding remote visits due to COVID-19 showed a similar treatment effect as the overall analysis.

Prespecified analyses of NSAA change from baseline in total scores by age at enrollment are shown in Fig. 2B for patients from age 4 to $\leq 6.0$ years and Fig. $2 \mathrm{C}$ for the $>6.0$ year age group. At Week 52 , the change from baseline in NSAA total score in the subgroup of patients with baseline age $\leq 6.0$ years (LS mean [SE]) was negative (worsened) in the placebo group (-1.0 [0.64]), whereas this score had increased slightly in the edasalonexent group (0.4 [0.47]) and the treatment group difference of 1.4 [0.78] had a $p$ value of 0.08 . In contrast, in the subgroup of patients with baseline age $>6.0$ years old, change from baseline in NSAA total score was negative at each time point throughout the study for both treatment groups, and no treatment group differences were observed $(p=0.89)$, (Table 2, Fig. 2C). The absolute NSAA 

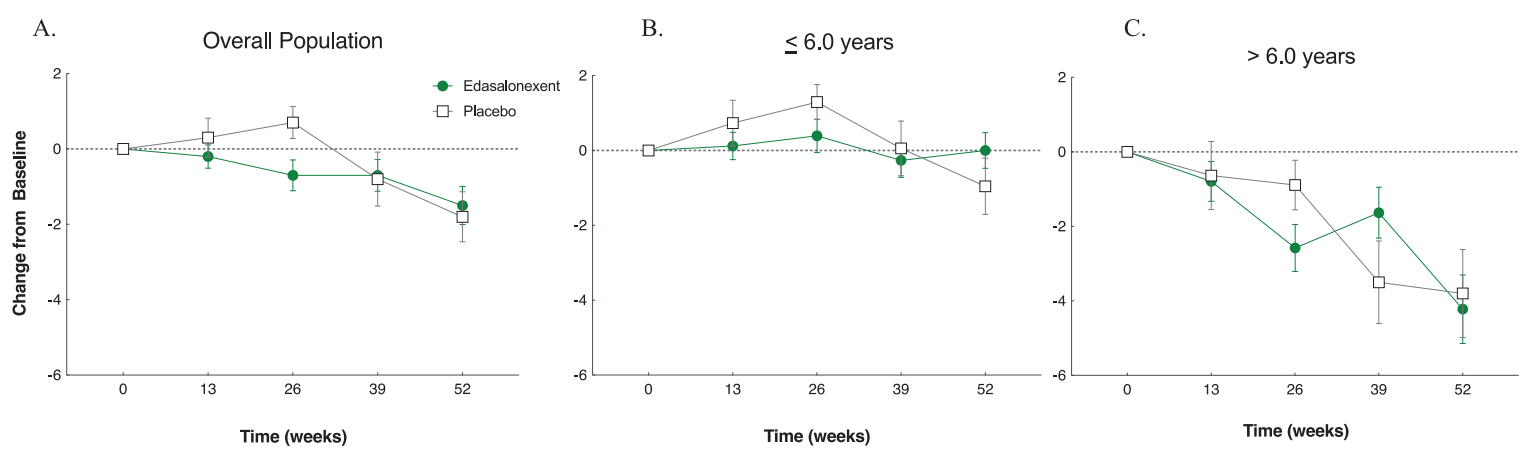

Fig. 2. NSAA Total Score (mean \pm SE) change from baseline overall population (A) and by age group for patients $\leq 6.0$ years (B) and $>6.0$ years $(\mathrm{C})$.

total scores for each age group are shown in Supplemental Figure 1S.

\section{Timed function tests}

Baseline TFTs (stand from supine, 10MRWT and 4-stair climb) speeds and changes from baseline at Week 52 for patients in the full analysis population are shown in Table 2. In the overall population, a comparison of changes from baseline between placebo and edasalonexent did not show statistically significant differences for any endpoint (Table 2), although there was consistently less worsening from baseline in the edasalonexent group as shown by change from baseline in speed and absolute times (Table 2 and Fig. 3).

While changes in outcome measures over the observation period were not statistically significant, favorable trends were identified. The change from baseline in stand from supine speed indicated worsening over time for both the edasalonexent and placebo groups, and there was a steeper decline in speed in the placebo group compared to the edasalonexent group (Fig. 3A). Time to stand from supine absolute times increased to a greater extent in the placebo group compared to the edasalonexent group (Fig. 3B). Similar trends were noted for the 4-stair climb and 10 meter walk/run speeds (Fig. 3C and 3E, respectively), where decline in speed over time was greater in the placebo versus edasalonexent groups. Time needed to complete the tasks was greater in the placebo group compared to the edasalonexent group (Fig. 3D and $3 \mathrm{~F})$.

The prespecified analyses of TFT speeds by age at enrollment are shown in Table 2 and Fig. 3. In the placebo group the times to complete all tasks increased (and corresponding speeds decreased) throughout the 52 weeks; declines in function were greatest in older patients. The greatest decline in function was seen for the time to stand from supine (Fig. 3A and B) and the 4-stair climb tests (Fig. 3C and D). For patients $\leq 6.0$ years old, there was less functional decline in the edasalonexent group over 52 weeks compared to the placebo group for all timed function tests (Fig. 3). For time to stand from supine speeds (Fig. 3A), LS mean (SE) change from baseline was $-0.014(0.008) / \mathrm{sec}$ in the edasalonexent group $\leq 6.0$ years compared to $-0.041(0.011) / \mathrm{sec}$ in the placebo group, and the treatment group difference was statistically significant $(-0.027 \quad[0.013] / \mathrm{sec}$; $p=0.046$ ) (Table 2). Similarly, for the 4-stair climb speed (Fig. 3C), patients $\leq 6.0$ years old in the edasalonexent group showed a slightly positive (improved) change from baseline in speed to complete the 4Stair Climb (0.001 [0.011]/sec) compared to the placebo group $(-0.043[0.016] / \mathrm{sec})$, and the treatment group difference was statistically significant $(0.044[0.019] / \mathrm{sec} ; p=0.021)$. For the change from baseline in 10 meter walk/run speed, treatment group differences did not reach statistical significance, although there was less decline in the edasalonexent group compared to placebo (Fig. 3E and F).

In contrast to the younger patient subgroup, those $>6.0$ years old treated with edasalonexent showed declines in function tests similar to placebo, and there was greater variability at each time point compared to the younger group.

\section{Performance of the upper limb}

At baseline, 74.6\% (85/114) of patients had a PUL entry score of 6 , (i.e., could abduct both arms simultaneously in extension in a full circle and touch above their head), while $25.4 \%$ (29/114) had an entry score of 5, (i.e., could raise their arms above head, but only by flexing their elbows). There was minimal change 
at the 52-week time point: $0.0(0.4)$ vs $0.1(0.4)$ in the edasalonexent and placebo group, respectively.

\section{PODCI}

At Week 52, a decrease (worsening) in the PODCI transfer and basic mobility score (LS mean [SE]) was observed in both the edasalonexent group $(-2.7$ [1.33]) and placebo group (-5.4 [1.72]), and the group difference was not statistically significant (2.7 [2.05]; $p=0.19)$. However, there was numerically less decline in the edasalonexent group compared to placebo. Figure 4A shows the individual item PODCI scores for the overall population. Changes in each of the PODCI individual scores were numerically more negative for the placebo group than the edasalonexent group including the PODCI transfer and basic mobility scale.
A.
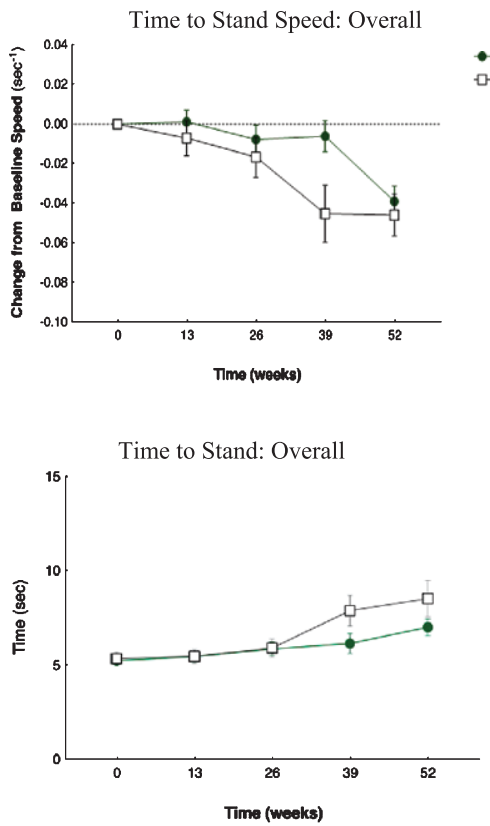

C.

4-Stair Climb Speed: Overall

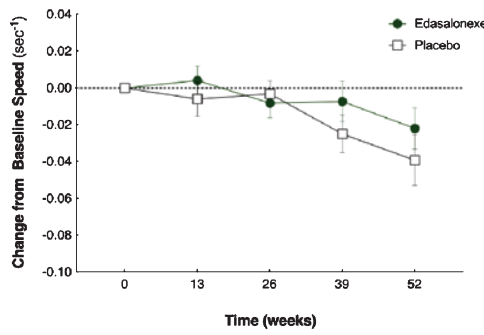

D.

4-Stair Climb Time: Overall

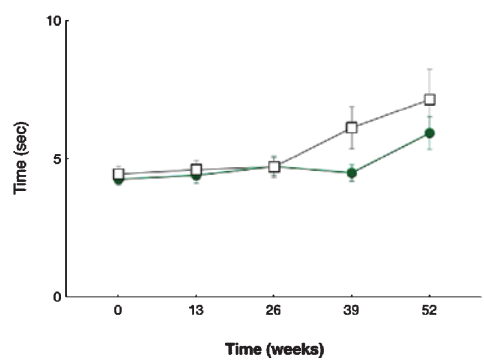

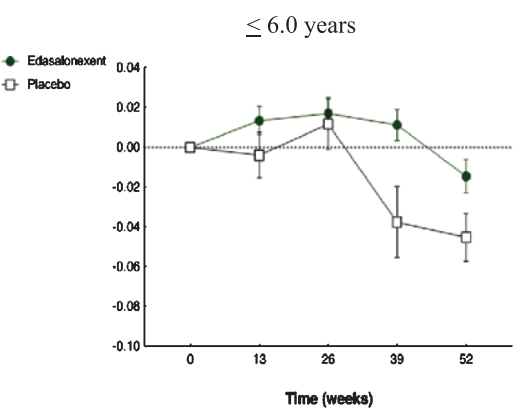

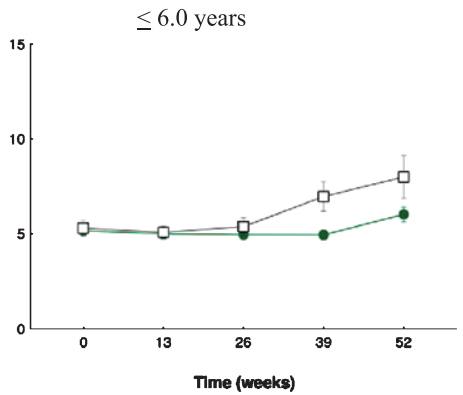

$\leq 6.0$ years

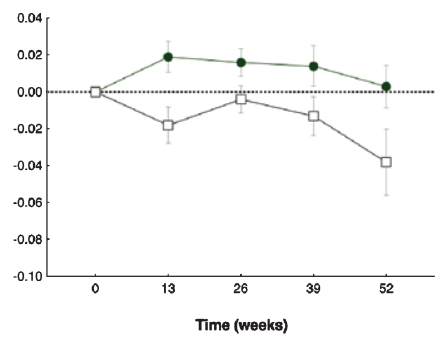

$\leq 6.0$ years
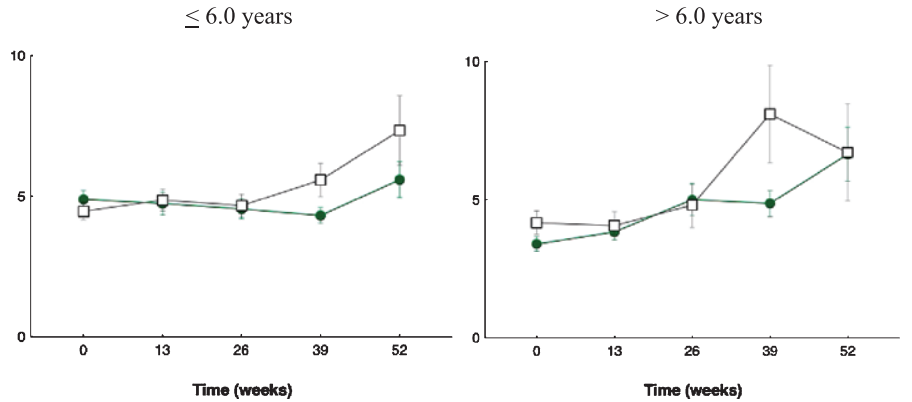

$>6.0$ years

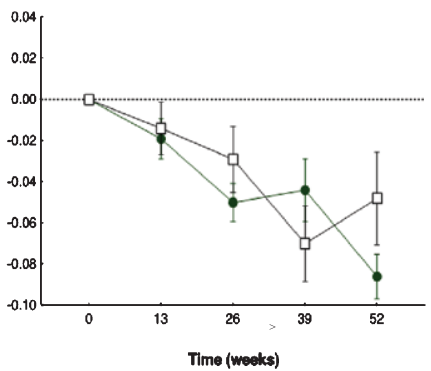

$>6.0$ years

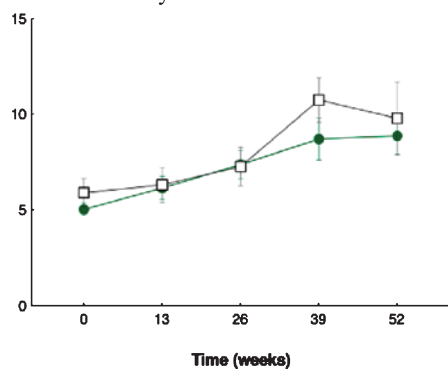

$>6.0$ years

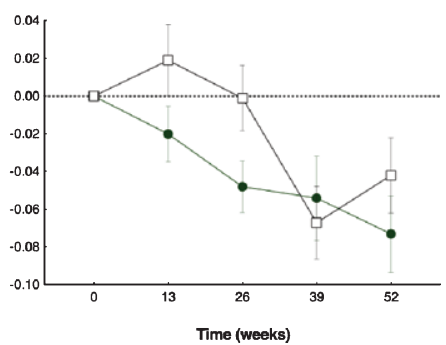

$>6.0$ years

Fig. 3. (Continued) 
E. 10-meter Run/Walk Speed: Overall

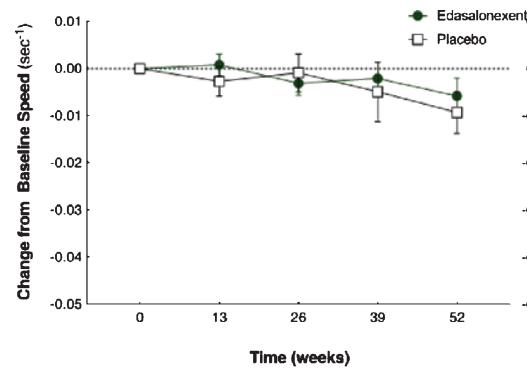

F.

10-meter Run/Walk Time: Overall

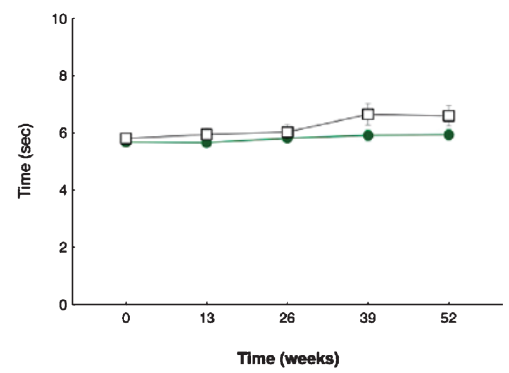

$\leq 6.0$ years

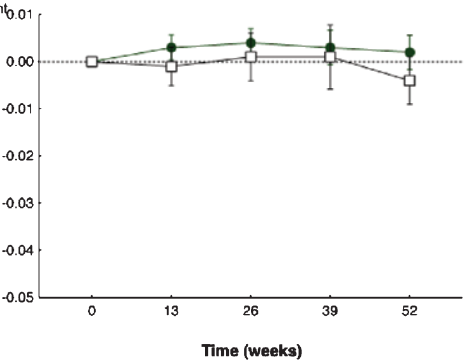

$\leq 6.0$ years

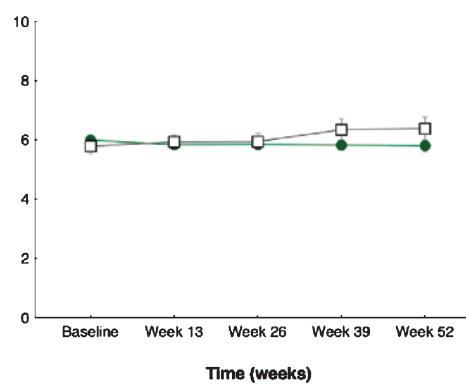

$>6.0$ years

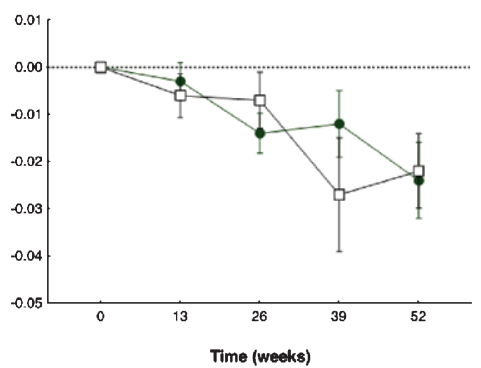

$>6.0$ years

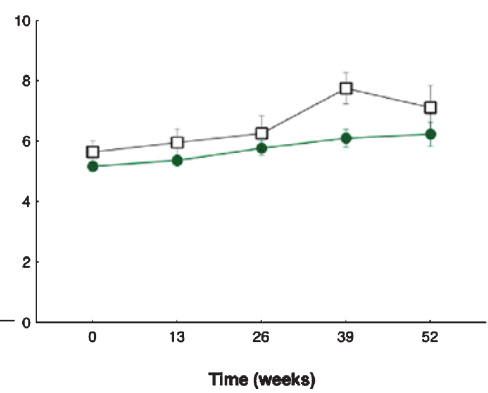

Fig. 3. Timed Function Assessments. Change from baseline (mean \pm SE) for timed function test speed ( $\mathrm{sec}^{-1}$ ) and the absolute times (sec) for the overall population and age groups patients $\leq 6.0$ years and $>6.0$ years. (A) Time to stand from supine speed change from baseline (B) Time to stand from supine absolute time C. 4-stair climb change from baseline D. 4-stair climb absolute time. E. 10MRWT speed change from baseline. F. 10MRWT absolute time.

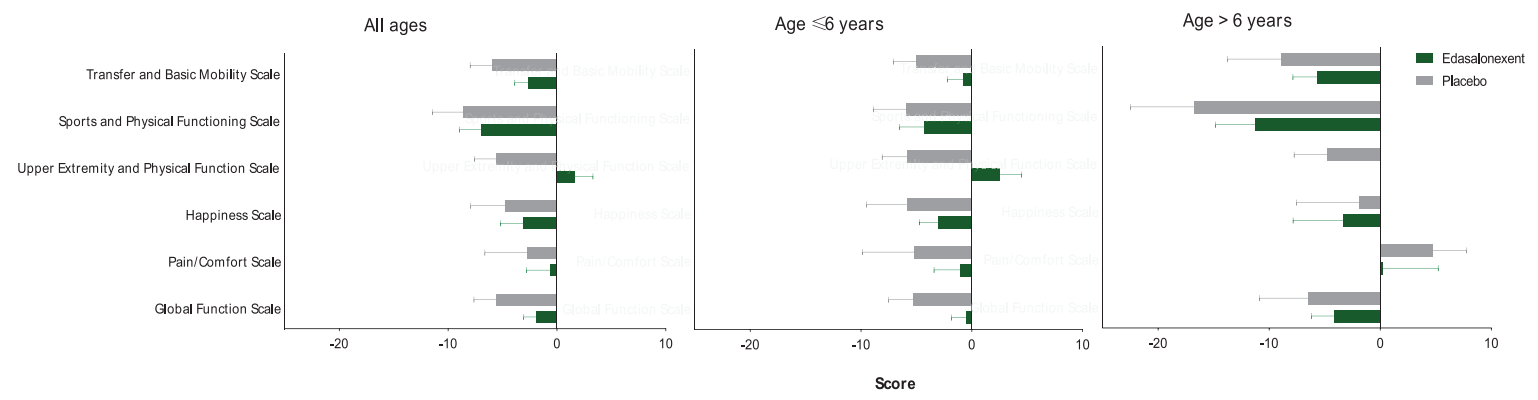

Fig. 4. PODCI Scales for the Overall Population and by Age Group for Patients $\leq 6.0$ years and $>6.0$ years.

For the subset of patients $\leq 6.0$ years old, changes from baseline (LS mean [SE]) in PODCI scores were negative (representing a worsening) in both the placebo $(-5.1[1.90])$ and edasalonexent $(-0.3$ [1.59]) groups. However, the change from baseline in the edasalonexent group was favorable compared to that of the placebo group, and the difference between treatment groups was statistically significant (4.9 [2.40]; $p=0.048$ ). Figure $4 \mathrm{~B}$ and $4 \mathrm{C}$ show the shows the individual item PODCI scores for patients $\leq 6.0$ and $>6.0$ years old, respectively. For the younger age group, changes in each of the PODCI individual scores were numerically more negative for the placebo group than the edasalonexent group including the PODCI transfer and basic mobility core scale, whereas for the older age group most but not all PODCI subscores were more negative for the placebo group than for the edasalonexent group.

\section{Safety and tolerability}

There were no adverse trends in vital signs, blood chemistry, hematology, coagulation, or measures of adrenal function over 52 weeks of treatment. 
Table 3

Profile of Treatment-Emergent Adverse Events

\begin{tabular}{|c|c|c|c|}
\hline & $\begin{array}{c}\text { Placebo } \\
N=43\end{array}$ & $\begin{array}{c}\text { Edasalonexent } \\
N=88 \\
100 \mathrm{mg} / \mathrm{kg} / \text { day }\end{array}$ & $\begin{array}{l}\text { Overall } \\
N=131\end{array}$ \\
\hline Treatment-Emergent Adverse Events & \# Patients $(\%)$ & & \\
\hline Any & $41(95.3)$ & $85(96.6)$ & $126(96.2)$ \\
\hline Mild & $31(72.1)$ & $53(60.2)$ & $84(64.1)$ \\
\hline Moderate & $9(20.9)$ & $31(35.2)$ & $40(30.5)$ \\
\hline Severe (both unrelated) & $1(2.3)$ & $1(1.1)$ & $2(1.5)$ \\
\hline Serious (both unrelated) & $1(2.3)$ & $1(1.1)$ & $2(1.5)$ \\
\hline Withdrawal due to Adverse Event & 0 & $1(1.1)$ & $1(0.8)$ \\
\hline \multicolumn{4}{|c|}{ Treatment-emergent events occurring in $>5 \%$ of patients } \\
\hline Diarrhea & $12(27.9)$ & $54(61.4)$ & $66(50.4)$ \\
\hline Vomiting & $11(25.6)$ & $29(33.0)$ & $40(30.5)$ \\
\hline Rash & $2(4.7)$ & $20(22.7)$ & $22(16.8)$ \\
\hline Nasopharyngitis & $9(20.9)$ & $19(21.6)$ & $28(21.4)$ \\
\hline Upper respiratory tract infection & $5(11.6)$ & $18(20.5)$ & $23(17.6)$ \\
\hline Pyrexia & $9(20.9)$ & $17(19.3)$ & $26(19.8)$ \\
\hline Abdominal pain upper & $9(20.9)$ & $15(17.0)$ & $24(18.3)$ \\
\hline Fall & $4(9.3)$ & $14(15.9)$ & $18(13.7)$ \\
\hline Cough & $11(25.6)$ & $13(14.8)$ & $24(18.3)$ \\
\hline Headache & $8(18.6)$ & $12(13.6)$ & $20(15.3)$ \\
\hline Influenza & $2(4.7)$ & $10(11.4)$ & $12(9.2)$ \\
\hline Muscle spasms & $1(2.3)$ & $10(11.4)$ & $11(8.4)$ \\
\hline Ear infection & $5(11.6)$ & $7(8.0)$ & $12(9.2)$ \\
\hline Nausea & $5(11.6)$ & $7(8.0)$ & $12(9.2)$ \\
\hline Pain in extremity & $5(11.6)$ & $6(6.8)$ & $11(8.4)$ \\
\hline Constipation & $3(7.0)$ & $6(6.8)$ & $9(6.9)$ \\
\hline Pharyngitis streptococcal & $4(9.3)$ & $4(4.5)$ & $8(6.1)$ \\
\hline Rhinorrhea & $5(11.6)$ & $3(3.4)$ & $8(6.1)$ \\
\hline Decreased appetite & $2(4.7)$ & $6(6.8)$ & $8(6.1)$ \\
\hline Abdominal pain & $1(2.3)$ & $7(8.0)$ & $8(6.1)$ \\
\hline Contusion & $2(4.7)$ & $6(6.8)$ & $8(6.1)$ \\
\hline Epistaxis & $1(2.3)$ & $6(6.8)$ & $7(5.3)$ \\
\hline Fatigue & $0(0.0)$ & $6(6.8)$ & $6(4.6)$ \\
\hline \multicolumn{4}{|c|}{ Treatment-related events occurring in $\geq 2.0 \%$ of patients } \\
\hline Any & $14(32.6)$ & $61(69.3)$ & $75(57.3)$ \\
\hline Diarrhea & $8(18.6)$ & $45(51.1)$ & $53(40.5)$ \\
\hline Vomiting & $2(4.7)$ & $14(15.9)$ & $16(12.2)$ \\
\hline Upper abdominal pain & $4(9.3)$ & $7(8.0)$ & $11(8.4)$ \\
\hline Rash & 0 & $9(10.2)$ & $9(6.9)$ \\
\hline Nausea & $1(2.3)$ & $4(4.5)$ & $5(3.8)$ \\
\hline Abdominal pain & 0 & $5(5.7)$ & $5(3.8)$ \\
\hline Abdominal discomfort & $1(2.3)$ & $3(3.4)$ & $4(3.1)$ \\
\hline Headache & 0 & $4(4.5)$ & $4(3.1)$ \\
\hline Fall & 0 & $3(3.4)$ & $3(2.3)$ \\
\hline Decreased appetite & 0 & $3(3.4)$ & $3(2.3)$ \\
\hline
\end{tabular}

ECG parameters remained similar to baseline values throughout the study, including resting heart rate, and no clinically significant ECG changes or abnormalities were noted during the study.

The adverse event profile for treatment-emergent and treatment-related adverse events for placebo and edasalonexent groups through 52 weeks is shown in Table 3. Incidence of events were similar between edasalonexent and placebo groups (96.6\% and 95.3\%, respectively). A serious event was reported in one patient in each group (norovirus in the patient in the edasalonexent group, and bronchilitis for the patient in the placebo group), neither of which were considered related to study drug, and both of which were considered to be severe in intensity. One patient in the edasalonexent group discontinued after experiencing a nonserious event of rash considered possibly related to treatment.

The most frequent events ( $\geq 2.0 \%$ of patients) related to treatment are shown in Table 3 . The incidence of study drug-related events was greater in the edasalonexent group (69.3\%) than in the placebo 
group (32.6\%). Among those reported more frequently (by a factor of 2 or more) in the edasalonexent group than in the placebo group were diarrhea ( $51.1 \%$ vs. $18.6 \%)$, vomiting ( $15.9 \%$ vs. $4.7 \%)$, rash $(10.2 \%$ and $0.0 \%)$, abdominal pain $(5.7 \%$ vs. $0.0 \%)$, headache ( $4.5 \%$ vs. $0.0 \%)$, fall ( $3.4 \%$ vs. $0.0 \%)$, and decreased appetite ( $3.4 \%$ vs. $0.0 \%$ ). Those occurring with similar frequency included upper abdominal pain and abdominal discomfort.

Patients in both groups had similar increases in height during the study: mean increase 6.04 (1.61) versus $6.33(1.74) \mathrm{cm}$ in the edasalonexent and placebo groups respectively. The mean weight gain at Week 52 was higher in the placebo group than the edasalonexent group [2.87 (1.97) $\mathrm{kg}$ and 0.85 (1.03) $\mathrm{kg}$, respectively] and impacted patient BMI. At baseline, patients in both groups had slightly above average mean BMI for their age $(65.9 \%$ and $68.3 \%$ for edasalonexent and placebo, respectively). By Week 52, patients in the edasalonexent group had a decline in mean BMI percentile of $-7.9 \%$ and a more appropriate BMI for their age (56.2\%), compared to patients in the placebo group who had a decline in BMI percentile of $-2.9 \%$ and a higher mean BMI percentile for their age $(65.8 \%)$.

\section{Post-hoc analyses of relationship of age to NSAA baseline and change}

Figure 5A shows the change in NSAA over 1 year stratified by age at enrollment. While there was a small increase in NSAA in patients aged 4 at baseline, those 5 years and older showed declines in total NSAA over 1 year. However, cross-sectional analysis of baseline NSAA as a function of age showed an overall positive correlation $(p<0.01)$. Indeed, the mean baseline NSAA of patients enrolled at age 6.0 was approximately 4 points greater than those enrolled at age 4 (Fig. 5B). Those enrolled at age 6 or 7 tended to have been diagnosed at an older age $(r=0.47, p<0.0001)$ and had a later reported onset of symptoms $(r=0.27, p=0.002)$.

\section{DISCUSSION}

Mutation-independent treatments for patients with DMD remains an unmet medical need. Since activation of NF- $\kappa \mathrm{B}$ occurs regardless of the underlying dystrophin mutation $[9,28,29]$, the NF- $\kappa$ B inhibitor edasalonexent was hypothesized to be a potential foundational therapy for DMD suitable for all patients
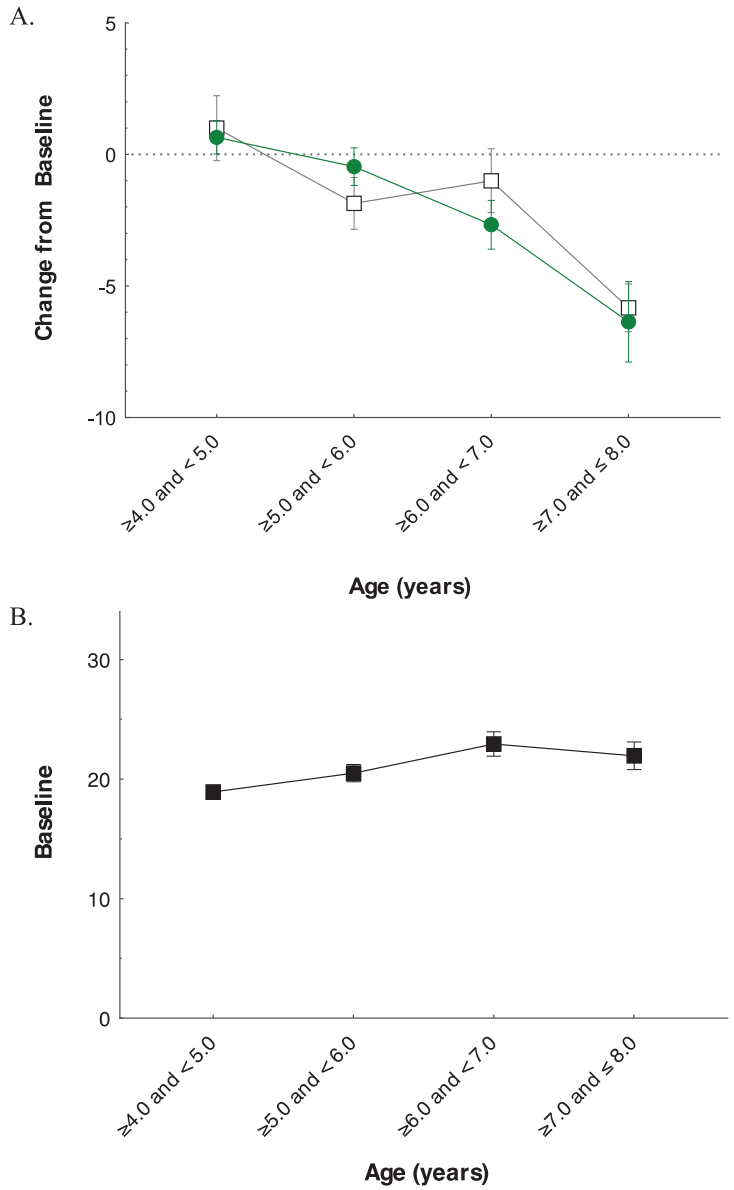

Fig. 5. Analysis of Impact of Baseline Age on NSAA Total Score. Change from baseline in total NSAA (mean \pm SE) at 52 weeks by age at enrollment (A), and baseline NSAA total score (mean \pm SD) by age at enrollment (B).

with DMD and compatible with use of exonskipping therapies. Edasalonexent was designed with the potential to limit muscle degeneration, promote muscle regeneration, and reduce inflammation and fibrosis to improve muscle function and thereby slow disease progression and muscle decline [4]. Stopping or slowing effects on muscle function is considered by parents/caregivers of patients with DMD as the most important outcome of DMD therapy [30].

In the present study, changes from baseline in the NSAA and functional assessments between the edasalonexent-treated and placebo-treated groups were not statistically significant in the overall population, although there were consistent trends showing less functional decline in patients treated with edasalonexent. In younger patients, statistically significant differences favoring edasalonexent were observed. 
After 52 weeks, changes from baseline in NSAA total score, standing from supine speed and 4-stair climb speed favored edasalonexent over placebo for patients 6.0 years of age and less, but not for older patients. For those receiving placebo, the rate of disease progression differed by age, with declines in functional assessments over 1 year more pronounced among older compared to younger patients. The PODCI showed trends favoring edasalonexent in scales related to function (such as transfer and basic mobility, sports and physical functioning, and upper extremity and physical function), and as with the timed function tests, trends were more robust in the younger patient group. Interestingly, a recent phase $1 / 2$ study of a plant flavonoid compound with NF$\kappa \mathrm{B}$ inhibitory properties, among other activities, in patients age 4-12 years with DMD found no differences in the NSAA between treated and untreated patients, but a trend for improvement in time to stand from supine in patients $<7$ years of age [31]. Thus, treatment with NF- $\kappa \mathrm{B}$ inhibitors in younger patients with DMD may warrant further study.

The study was designed to be a well-controlled evaluation of the effects of edasalonexent in a significant, already-existent population of DMD patients not on steroids at this stage of their disease. While enrollment criteria required patients to have been off steroids for least 24 weeks, most (97\%) were steroidnaïve. In this age group at the early ambulatory stage of the disease, a substantial number of boys who have been diagnosed with DMD are not being treated with corticosteroids. In both the US and Europe, over 30\% of boys in this age range were reported not to be on steroids, in some cases because parents declined or deferred treatment $[32,33]$. The protocol guided investigators to consider the clinical trajectory of patients at enrollment and as they continued in the trial and to recommend transition to steroids if they felt that clinical progression warranted withdrawal and steroid initiation.

Inclusion of a steroid arm in addition to the placebo arm for registration purposes of edasalonexent was considered, but there were significant barriers to a well-controlled study with a steroid comparator arm, including the inability to adequately blind the study and the uncertainty about the duration of exposure to appropriately power a study for edasalonexent vs steroids. The intent was that head-to-head study would be part of the subsequent development plan; however, the duration of that study would likely have to have been much longer, which would have precluded the ability to include a placebo arm.
While the functional status of those with DMD in the age range from 4 to 7 on steroids is generally acknowledged to be stable or improved as assessed by the NSAA [25, 34], patients in this trial generally declined over the 52 weeks, consistent with observations in the off-treatment period in the Phase 2 study of edasalonexent in steroid-naive patients [22]. To our knowledge, the placebo group in the current study represents the largest observational study of the NSAA in patients aged 4 to 7 not on steroids.

Compared to expected outcomes in DMD where older patients not on steroids would have lower functional status at baseline and a more rapid longitudinal decline when compared to younger patients, the older population in our study had higher baseline NSAA scores than the younger group. The paradox of the higher baseline scores coupled with the observed negative NSAA trajectory suggests a potential difference in disease severity in the largely steroid-naïve older versus younger patients and does not support a maturational improvement in this population. To better understand this observation, we looked at baseline characteristics of the two age groups. Those enrolled at ages 6 or 7 were diagnosed at an older age and had later onset of symptoms compared to patients less than 6 years, suggesting a less severe phenotype in the older group consistent with their higher NSAA at baseline. It is likely that patients with earlier onset of disease have earlier onset of functional deficits, so there appears to be a selection bias for patients who remain steroid naïve over age 6 to have a milder disease phenotype. While it is generally considered that targeting patients less than 8 years of age for treatment is considered optimal in order to prevent irreversible muscle damage, our study suggests that there may be heterogeneity in disease characteristics and progression within the younger steroid-naïve age group. Understanding of baseline characteristics to ensure a less variable population of DMD patients and appropriateness of endpoints may help in design of future DMD trials.

The efficacy endpoints selected were chosen since they reflect functional assessments that have significant impact on patients. The NSAA is a validated instrument that has undergone detailed psychometric evaluations based on traditional (reliability and validity) and modern (Rasch analysis) methods [35, 36], and it has been included in multiple international clinical trials. In addition, up to 36-month longitudinal data are available in patients less than 8 years of age with DMD [25, 34, 37-39]. For patients in the placebo group, the declines over 52 weeks in the time-to-stand 
and 4-stair-climb timed function tests were similar to those observed the phase 2 study during off-treatment periods, while declines in the NSAA and 10MRWT were less than those observed in the phase 2 study [22]. Enrollment criteria and patient baseline characteristics for both studies were similar. Other than the heterogeneity of the patient population when stratified by age, it is unclear why the results of this phase 3 study did not show greater stabilization or improvement in functional endpoints that were indicated by the phase 2 study analyses [22]. A potential limitation of the phase 3 study design was reliance on information collected during the off-treatment period in the phase 2 study to power this phase 3 study, but which did not appear to be replicated by the placebo arm of the current study. In the phase 2 study, there was a smaller number of patients and a shorter observation period, and extrapolation was used in the design of this phase 3 study.

Edasalonexent has been well tolerated and without serious adverse events or dose reductions during phase 1 studies [20] in adults, and the phase 1/2 study in pediatric patients with DMD [21]. This was confirmed in the phase 3 study through 52 weeks of treatment. Baseline laboratory and ECG abnormalities, such as resting tachycardia, were consistent with clinical observations in patients with DMD [40, 41] and were not changed following edasalonexent treatment. The most common adverse events were GI disturbances. Mean height increases during the study were similar between groups, while the mean weight gain at Week 52 was lower in the edasalonexent group with resulting decrease in mean BMI percentile and a more appropriate BMI for age compared to the placebo group. The interpretations of the analyses by age group are limited by the small numbers of patients in each group, especially in the older age group.

While challenges arose during the study due to the COVID-19 pandemic, overall compliance with study drug remained high and there were no patient discontinuations due to COVID-19 travel restrictions or illness. Most 52-week visits took place during the pandemic, with only $15 \%$ of visits conducted remotely. In remote visits, only NSAA and time to stand from supine could be consistently reported since standard equipment and space for other timed function tests precluded assessment. Nonetheless, sensitivity analysis of change from baseline in NSAA excluding remote visits showed a similar treatment effect as the overall analysis, from which we conclude that COVID-19 did not alter the key study results. However, several patients had visit -to-visit variations in some measures that raised questions regarding the validity of remote assessments. Efforts to validate remote versus clinic visits are needed.

Because of saturable absorption of edasalonexent [20] increasing the dose was not feasible to increase exposure. Nonetheless, given trends in efficacy, particularly in the younger patients, we believe that the $\mathrm{NF}-\kappa \mathrm{B}$ pathway may still be an appropriate target for therapeutic intervention in DMD.

\section{CONCLUSIONS}

Edasalonexent was generally well tolerated with a manageable safety profile at the dose of $100 \mathrm{mg} /$ $\mathrm{kg} / \mathrm{day}$. Although edasalonexent did not achieve statistical significance for improvement in primary and secondary functional endpoints for assessment of DMD in the overall population, subgroup analysis suggested that edasalonexent may slow disease progression in younger patients.

\section{DECLARATIONS OF INTEREST}

RSF, CMM, EF, ENK, KM, WM, JS, JN, HM, GM, CT, MR, DOR, WMF, MT, WBB, CTN, KV, JJ, HP and all additional members of the Polaris study group are principal investigators in clinical trials for Catabasis and have received research support from Catabasis. HLS, ME and KW have received research support from Catabasis. RSF, CMM, HLS, and ME are consultants for Catabasis. MM, JM, and JMD were employees/contractors of Catabasis Pharmaceuticals, Inc. at the time of the study. All authors participated in the conduct of the study as well as manuscript planning, preparation and editing. In addition, RSF, CMM, HLS, MM, JM and JMD participated in the design and analysis of the study. All authors approved the final manuscript.

\section{FUNDING}

Catabasis Pharmaceuticals, Inc. provided financial support for the conduct of the study and data analyses, and for the preparation of the manuscript.

\section{ACKNOWLEDGMENTS}

The authors thank the subjects and their families for their participation, and the staff of the clinical research organizations at each study site, including study coordinators, clinical evaluators, 
co-investigators, and pharmacists, for conduct of the studies, the clinical assessors of ATOM International, and gratefully acknowledge colleagues at Catabasis Pharmaceuticals, Inc. for their contributions to the development of edasalonexent. The list of Polaris DMD Study Group members, Study Coordinators and Clinical Evaluators are provided in Supplementary Material. The authors thank the members of the Data Safety Monitoring Board: Anne Connolly, M.D., Craig Campbell, M.D., Susan Iannaccone, M.D., William McDermott, M.D., and Brian Denger. All authors participated in the design, conduct, and analysis of the study, and manuscript development and approved the final manuscript. Catabasis Pharmaceuticals, Inc. provided financial support for the conduct of the research and data analyses. Patrice $C$. Ferriola, PhD, of KZE PharmAssociates, LLC provided medical writing assistance and was funded by Catabasis Pharmaceuticals, Inc.

\section{SUPPLEMENTARY MATERIAL}

The supplementary material is available in the electronic version of this article: https://dx.doi.org/ $10.3233 / \mathrm{JND}-210689$.

\section{REFERENCES}

[1] Mah JK, Korngut L, Dykeman J, Day L, Pringsheim T, Jette N. A systematic review and meta-analysis on the epidemiology of Duchenne and Becker muscular dystrophy. Neuromuscul Disord. 2014;24:482-91.

[2] Kieny P, Chollet S, Delalande P, Le Fort M, Magot A, Pereon Y, et al. Evolution of life expectancy of patients with Duchenne muscular dystrophy at AFM Yolaine de Kepper centre between 1981 and 2011. Ann Phys Rehabil Med. 2013;56:443-54.

[3] Matthews E, Brassington R, Kuntzer T, Jichi F, Manzur AY. Corticosteroids for the treatment of Duchenne muscular dystrophy. Cochrane Database Syst Rev. 2016:Cd003725.

[4] Hammers DW, Sleeper MM, Forbes SC, Coker CC, Jirousek MR, Zimmer M, et al. Disease-modifying effects of orally bioavailable NF-kappaB inhibitors in dystrophin-deficient muscle. JCI Insight. 2016;1:e90341.

[5] Kornegay JN, Peterson JM, Bogan DJ, Kline W, Bogan JR, Dow JL, et al. NBD delivery improves the disease phenotype of the golden retriever model of Duchenne muscular dystrophy. Skelet Muscle. 2014;4:18.

[6] Kumar A, Boriek AM. Mechanical stress activates the nuclear factor-kappaB pathway in skeletal muscle fibers: a possible role in Duchenne muscular dystrophy. FASEB J. 2003; 17:386-96.

[7] Rosenberg AS, Puig M, Nagaraju K, Hoffman EP, Villalta SA, Rao VA, et al. Immune-mediated pathology in Duchenne muscular dystrophy. Sci Transl Med. 2015;7: 299 rv4.
[8] Shin J, Tajrishi MM, Ogura Y, Kumar A. Wasting mechanisms in muscular dystrophy. Int J Biochem Cell Biol. 2013;45:2266-79.

[9] Acharyya S, Villalta SA, Bakkar N, Bupha-Intr T, Janssen PM, Carathers $M$, et al. Interplay of IKK/NF-kappaB signaling in macrophages and myofibers promotes muscle degeneration in Duchenne muscular dystrophy. J Clin Invest. 2007;117:889-901.

[10] Li H, Malhotra S, Kumar A. Nuclear factor-kappa B signaling in skeletal muscle atrophy. J Mol Med (Berl). 2008; 86:1113-26.

[11] Peterson JM, Bakkar N, Guttridge DC. NF-kappaB signaling in skeletal muscle health and disease. Curr Top Dev Biol. 2011;96:85-119.

[12] Hu X, Blemker S. Musculoskeletal simulation can help explain selective muscle degeneration in Duchenne muscular dystrophy. Muscle Nerve. 2015;52:174-82.

[13] Porter JD, Merriam AP, Leahy P, Gong B, Khanna S. Dissection of temporal gene expression signatures of affected and spared muscle groups in dystrophin-deficient $(\mathrm{mdx})$ mice. Hum Mol Genet. 2003;12:1813-21.

[14] Forbes SC, Willcocks RJ, Triplett WT, Rooney WD, Lott DJ, Wang DJ, et al. Magnetic resonance imaging and spectroscopy assessment of lower extremity skeletal muscles in boys with Duchenne muscular dystrophy: a multicenter cross sectional study. PLoS One. 2014;9:e106435.

[15] Vu CB, Bemis JE, Benson E, Bista P, Carney D, Fahrner R, et al. Synthesis and Characterization of Fatty Acid Conjugates of Niacin and Salicylic Acid. J Med Chem. 2016;59: 1217-31.

[16] Williams-Bey Y, Boularan C, Vural A, Huang NN, Hwang IY, Shan-Shi C, et al. Omega-3 free fatty acids suppress macrophage inflammasome activation by inhibiting NFkappaB activation and enhancing autophagy. PLoS One. 2014;9:e97957.

[17] Zwart SR, Pierson D, Mehta S, Gonda S, Smith SM. Capacity of omega-3 fatty acids or eicosapentaenoic acid to counteract weightlessness-induced bone loss by inhibiting NF-kappaB activation: from cells to bed rest to astronauts. J Bone Miner Res. 2010;25:1049-57.

[18] Kopp E, Ghosh S. Inhibition of NF-kappa B by sodium salicylate and aspirin. Science. 1994;265:956-9.

[19] Yin MJ, Yamamoto Y, Gaynor RB. The anti-inflammatory agents aspirin and salicylate inhibit the activity of I(kappa)B kinase-beta. Nature. 1998;396:77-80.

[20] Donovan JM, Zimmer M, Offman E, Grant T, Jirousek M. A Novel NF-kappaB Inhibitor, Edasalonexent (CAT1004), in Development as a Disease-Modifying Treatment for Patients With Duchenne Muscular Dystrophy: Phase 1 Safety, Pharmacokinetics, and Pharmacodynamics in Adult Subjects. J Clin Pharmacol. 2017;57:627-639.

[21] Finanger E, Vandenborne K, Finkel RS, Lee Sweeney H, Tennekoon G, Yum S, et al. Phase 1 Study of Edasalonexent (CAT-1004), an Oral NF-kappaB Inhibitor, in Pediatric Patients with Duchenne Muscular Dystrophy. J Neuromuscul Dis. 2019;6:43-54.

[22] Finkel RS, Finanger E, Vandenborne K, Sweeney HL, Tennekoon G, Shieh PB, et al. Disease- modifying effects of edasalonexent, an $\mathrm{NF}-\kappa \mathrm{B}$ inhibitor, in young boys with Duchenne muscular dystrophy: $R$ esults of the MoveDMD phase 2 and open label extension trial. Neuromuscul Disord 2021;31(5):385-396. https://doi.org/10.1016/j.nmd.2021. 02.001 .

[23] Scott E, Eagle M, Mayhew A, Freeman J, Main M, Sheehan J, et al. Development of a functional assessment scale 
for ambulatory boys with Duchenne muscular dystrophy. Physiother Res Int. 2012;17:101-9.

[24] Henricson E, Abresch R, Han JJ, Nicorici A, Goude Keller E, de Bie E, et al. The 6-Minute Walk Test and PersonReported Outcomes in Boys with Duchenne Muscular Dystrophy and Typically Developing Controls: Longitudinal Comparisons and Clinically-Meaningful Changes Over One Year. PLoS Curr. 2013;5.

[25] Coratti G, Brogna C, Norcia G, Ricotti V, Abbott L, D'Amico A, et al. Longitudinal natural history in young boys with Duchenne muscular dystrophy. Neuromuscul Disord. 2019;29:857-62.

[26] Bushby K, Connor E. Clinical outcome measures for trials in Duchenne muscular dystrophy: report from International Working Group meetings. Clinical investigation. 2011;1:1217-35.

[27] Mazzone E, Martinelli D, Berardinelli A, Messina S, D'Amico A, Vasco G, et al. North Star Ambulatory Assessment, 6-minute walk test and timed items in ambulant boys with Duchenne muscular dystrophy. Neuromuscul Disord. 2010;20:712-6.

[28] Archer SK, Garrod R, Hart N, Miller S. Dysphagia in Duchenne muscular dystrophy assessed by validated questionnaire. Int J Lang Commun Disord. 2013;48:240-6.

[29] Mourkioti F, Kratsios P, Luedde T, Song YH, Delafontaine P, Adami R, et al. Targeted ablation of IKK2 improves skeletal muscle strength, maintains mass, and promotes regeneration. J Clin Invest. 2006;116:2945-54.

[30] Peay HL, Hollin I, Fischer R, Bridges JF. A communityengaged approach to quantifying caregiver preferences for the benefits and risks of emerging therapies for Duchenne muscular dystrophy. Clin Ther. 2014;36:624-37.

[31] Vita GL, Sframeli M, Licata N, Bitto A, Romeo S, Frisone F, et al. A Phase 1/2 Study of Flavocoxid, an Oral NFkappaB Inhibitor, in Duchenne Muscular Dystrophy. Brain Sci 2021;11.

[32] Cowen L, Mancini M, Martin A, Lucas A, Donovan JM. Variability and trends in corticosteroid use by male United States participants with Duchenne muscular dystrophy in the Duchenne Registry. BMC Neurol. 2019;19:84.
[33] Landfeldt E, Lindgren P, Bell CF, Schmitt C, Guglieri M, Straub V, et al. Compliance to Care Guidelines for Duchenne Muscular Dystrophy. J Neuromuscul Dis. 2015;2:63-72.

[34] Miller NF, Alfano LN, Iammarino MA, Connolly AM, Moore-Clingenpeel M, Powers BR, et al. Natural History of Steroid-Treated Young Boys With Duchenne Muscular Dystrophy Using the NSAA, 100m, and Timed Functional Tests. Pediatr Neurol. 2020;113:15-20.

[35] Mayhew A, Cano S, Scott E, Eagle M, Bushby K, Muntoni F, et al. Moving towards meaningful measurement: Rasch analysis of the North Star Ambulatory Assessment in Duchenne muscular dystrophy. Dev Med Child Neurol. 2011;53:535-42.

[36] Mayhew AG, Cano SJ, Scott E, Eagle M, Bushby K, Manzur A, et al. Detecting meaningful change using the North Star Ambulatory Assessment in Duchenne muscular dystrophy. Dev Med Child Neurol. 2013;55:1046-52.

[37] Mazzone E, Vasco G, Sormani MP, Torrente Y, Berardinelli A, Messina S, et al. Functional changes in Duchenne muscular dystrophy: a 12-month longitudinal cohort study. Neurology. 2011;77:250-6.

[38] Pane M, Mazzone ES, Sivo S, Sormani MP, Messina S, D'Amico A, et al. Long term natural history data in ambulant boys with Duchenne muscular dystrophy: 36-month changes. PLoS One. 2014;9:e108205.

[39] Ricotti V, Ridout DA, Pane M, Main M, Mayhew A, Mercuri E, et al. The NorthStar Ambulatory Assessment in Duchenne muscular dystrophy: considerations for the design of clinical trials. J Neurol Neurosurg Psychiatry. 2016;87:149-55.

[40] Takami Y, Takeshima Y, Awano H, Okizuka Y, Yagi M, Matsuo M. High incidence of electrocardiogram abnormalities in young patients with duchenne muscular dystrophy. Pediatr Neurol. 2008;39:399-403.

[41] McMillan HJ, Gregas M, Darras BT, Kang PB. Serum transaminase levels in boys with Duchenne and Becker muscular dystrophy. Pediatrics. 2011;127:e132-6. 